\title{
Impact of age at diagnosis and duration of type 2 diabetes on mortality in Australia 1997-2011
}

\author{
Lili Huo $^{1,2}$ • Dianna J. Magliano ${ }^{2,3} \cdot$ Fanny Rancière ${ }^{4,5}$ - Jessica L. Harding ${ }^{2}$ - Natalie Nanayakkara ${ }^{3}$. \\ Jonathan E. Shaw ${ }^{2,3}$ • Bendix Carstensen ${ }^{6}$
}

Received: 26 July 2017 / Accepted: 27 November 2017 / Published online: 22 February 2018

(C) Springer-Verlag GmbH Germany, part of Springer Nature 2018

\begin{abstract}
Aims/hypothesis Current evidence suggests that type 2 diabetes may have a greater impact on those with earlier diagnosis (longer duration of disease), but data are limited. We examined the effect of age at diagnosis of type 2 diabetes on the risk of all-cause and cause-specific mortality over 15 years.

Methods The data of 743,709 Australians with type 2 diabetes who were registered on the National Diabetes Services Scheme (NDSS) between 1997 and 2011 were examined. Mortality data were derived by linking the NDSS to the National Death Index. All-cause mortality and mortality due to cardiovascular disease (CVD), cancer and all other causes were identified. Poisson regression was used to model mortality rates by sex, current age, age at diagnosis, diabetes duration and calendar time.

Results The median age at registration on the NDSS was 60.2 years (interquartile range [IQR] 50.9-69.5) and the median followup was 7.2 years (IQR 3.4-11.3). The median age at diagnosis was 58.6 years (IQR 49.4-67.9). A total of 115,363 deaths occurred during 7.20 million person-years of follow-up. During the first 1.8 years after diabetes diagnosis, rates of all-cause and cancer mortality declined and CVD mortality was constant. All mortality rates increased exponentially with age. An earlier diagnosis of type 2 diabetes (longer duration of disease) was associated with a higher risk of all-cause mortality, primarily driven by CVD mortality. A 10 year earlier diagnosis (equivalent to 10 years' longer duration of diabetes) was associated with a 1.2-1.3 times increased risk of all-cause mortality and about 1.6 times increased risk of CVD mortality. The effects were similar in men and women. For mortality due to cancer (all cancers and colorectal and lung cancers), we found that earlier diagnosis of type 2 diabetes was associated with lower mortality compared with diagnosis at an older age.

Conclusions/interpretation Our findings suggest that younger-onset type 2 diabetes increases mortality risk, and that this is mainly through earlier CVD mortality. Efforts to delay the onset of type 2 diabetes might, therefore, reduce mortality.
\end{abstract}

Keywords Age of onset $\cdot$ Cardiovascular disease $\cdot$ Cause of death $\cdot$ Diabetes $\cdot$ Diabetes onset $\cdot$ Diagnosis $\cdot$ Duration $\cdot$ Mortality

Jonathan E. Shaw and Bendix Carstensen are joint senior authors.

Lili Huo and Dianna J. Magliano are joint first authors.

Electronic supplementary material The online version of this article (https://doi.org/10.1007/s00125-018-4544-z) contains peer-reviewed but unedited supplementary material, which is available to authorised users.

Dianna J. Magliano

Dianna.Magliano@baker.edu.au

Bendix Carstensen

bendix.carstensen@regionh.dk

1 Department of Endocrinology, Beijing Jishuitan Hospital, Beijing, People's Republic of China

2 Department of Clinical Diabetes and Epidemiology, Baker Heart and Diabetes Institute, Level 4, 99 Commercial Road,

Melbourne, VIC 3004, Australia
3 Department of Epidemiology and Preventive Medicine, School of Public Health and Preventive Medicine, Monash University, Melbourne, VIC, Australia

4 Inserm, U1153, Epidemiology and Biostatistics Sorbonne Paris Cité Research Centre, Villejuif, France

5 Paris Descartes University, Sorbonne Paris Cité, UMR1153, Paris, France

6 Clinical Epidemiology, Steno Diabetes Center Copenhagen, Gentofte, Denmark 


\section{Research in context}

\section{What is already known about this subject?}

- The proportion of younger people with diabetes is increasing in many places in the world

\section{What is the key question?}

- What is the impact of age at diagnosis of diabetes on all-cause and cause-specific mortality rates?

\section{What are the new findings?}

- We show that a younger age at diagnosis of type 2 diabetes is associated with higher all-cause, cardiovascular disease (CVD), ischaemic heart disease and stroke mortality, but slightly lower cancer mortality (all cancers and lung and colorectal cancers) compared with diagnosis at an older age

- Thus, for two men of the same age but 10 years' difference in diabetes duration, the one with the earlier onset (and hence the longer duration of diabetes) has an approximately $30 \%$ and $60 \%$ greater risk of death from any cause and from CVD, respectively, relative to the person with the shorter duration

\section{How might this impact on clinical practice in the foreseeable future?}

- Our study suggests that early and aggressive CVD risk factor management is warranted for young-onset type 2 diabetes

$\begin{array}{ll}\text { Abbreviations } \\ \text { CVD } & \text { Cardiovascular disease } \\ \text { IHD } & \text { Ischaemic heart disease } \\ \text { IQR } & \text { Interquartile range } \\ \text { NDSS } & \text { National Diabetes Services Scheme } \\ \text { TODAY } & \text { Treatment Options for Type } 2 \text { Diabetes in } \\ & \text { Adolescents and Youth }\end{array}$

\section{Introduction}

Type 2 diabetes was once considered as a disease of middle-aged and older people, but in recent decades the prevalence of type 2 diabetes in adolescents and young adults has risen considerably around the world, driven largely by increasing obesity prevalence at younger ages. A study from Japan reported that type 2 diabetes increased tenfold in young children (aged 6-12 years) and doubled among adolescents over a 20 year period from 1976 to 1997 [1]. In Australia, those diagnosed with type 2 diabetes at age 10-39 years accounted for around 9\% of all new cases in the year 2011 [2]. Similar trends have been found in many countries across the world [3-5], making young adults the fastest growing group for newonset type 2 diabetes. Individuals diagnosed as having type 2 diabetes at a younger age seem to have an increased risk of developing diabetes-related complications compared with those diagnosed later in life, suggesting a more aggressive phenotype [6,7]. They also possess a clustering of adverse cardiovascular risk factors at an earlier age. The Treatment Options for Type 2 Diabetes in Adolescents and Youth (TODAY) study $[6,7]$ and the
SEARCH for Diabetes in Youth study $[8,9]$ have both demonstrated that obesity, dyslipidaemia, hypertension and microalbuminuria are common in youth with type 2 diabetes and worsen over time. Hillier and Pedula found that a diagnosis of type 2 diabetes under 45 years of age was associated with a 14-fold increase in the risk of myocardial infarction compared with control individuals without diabetes, which was substantially greater than the fourfold increase in those diagnosed over 45 years of age [10].

In parallel with advances in diabetes care, several recent studies have found a decline over time in the excess risk of death in people with type 2 diabetes [11, 12]. Nonetheless, a study from Sweden reported that younger people with type 2 diabetes (age $<55$ years) who had an $\mathrm{HbA}_{1 \mathrm{c}}$ level of $6.9 \%(52 \mathrm{mmol} / \mathrm{mol})$ or less still had a risk of death that was twice as high as that of the general population [13]. To date, only limited data are available for the impact of age at diagnosis on excess mortality in people with type 2 diabetes. A recent study from Denmark [11] showed that at any given age, younger age at diagnosis of type 2 diabetes was associated with higher mortality for men, but the excess risk attributable to age at diagnosis was very weak among women. This study did not examine the impact of age at diagnosis by different causes of death, which is necessary to help direct clinical interventions to prevent or reduce premature morbidity and mortality in type 2 diabetes. We therefore examined in this study the impact of age at diagnosis of type 2 diabetes on the risk of all-cause and cause-specific mortality over 15 years using a large national registry of type 2 diabetes in Australia. 


\section{Methods}

Population The National Diabetes Services Scheme (NDSS) was established in Australia in 1987 to deliver diabetes-related products at subsidised prices and provide information to people with diabetes. Registration of patients is carried out by a medical practitioner or certified diabetes educator. The NDSS captures $80-90 \%$ of all Australians with known diabetes [14]. In total, 1,108,420 people were registered with type 2 diabetes on the NDSS between 1997 and 2011. The primary population for this analysis included 744,188 (67.1\%) people with type 2 diabetes who had a date of diagnosis of diabetes listed on the NDSS. A secondary analysis included all type 2 diabetes registrants and used date of registration as a proxy for those whose date of diagnosis was missing (1,108,420 people).

Although the NDSS was initially established in 1987, 1997 was chosen as the start date, as this time period followed a unification of state-based registries and consequently an improvement in data quality. Diabetes type is classified by the health practitioner who completes the registration. For the current analysis, type 2 diabetes was assigned to all registrants who were classified as type 2 on the NDSS. Type 1 diabetes status was assigned to registrants who were recorded as type 1 on the NDSS registry, were registered at $<45$ years of age and were taking insulin. We chose 45 years as the cut-off to minimise the number of people with type 1 diabetes that we would miss without misclassifying significant numbers of people with type 2 diabetes as type 1 diabetes. In addition, registrants who were recorded as having type 2 diabetes on the registry and who were diagnosed before the age of 30 years and were taking insulin within 1 year of diagnosis were reclassified as having type 1 diabetes. All others were classified as having type 2 diabetes.

The NDSS was linked to the National Death Index up to 31 December 2011 by the Australian Institute of Health and Welfare as reported previously $[15,16]$. The underlying cause of death was coded according to ICD-10 (www. who.int/classifications/icd/en). In the primary analysis, causes of death were classified as follows: cardiovascular disease (CVD) I20-I25, I60-I69 or 'diabetes with circulatory complications' (E10.5, E11.5, E12.5, E13.5, E14.5), cancer $\mathrm{C} 00-\mathrm{C} 97$ and other underlying causes of death (non-CVD and non-cancer). We also explored mortality from ischaemic heart disease (IHD) (I20-I25), stroke (I60-I69), pancreatic cancer (C25-25.9), lung cancer (C33-C35.9) and colorectal cancer (C18-C20.9), since they are among those with the highest cancer death rates in individuals with diabetes in Australia. Deaths with an underlying cause corresponding to 'uncomplicated diabetes' (E10.9, E11.9, E12.9, E13.9, E14.9) where a CVD, IHD or stroke ICD code also appeared in the first line of part I on the death certificate were also included in the respective group. We considered these deaths as due to
CVD, IHD or stroke, as it is not possible to die from 'uncomplicated diabetes'. The NDSS dataset linked to the NDI has previously been used to present age-specific mortality rates among those with diabetes [17].

Statistical analysis and reporting Individuals were followed from 1 January 1997, or registration date if later, to 31 December 2011 or date of death, whichever occurred first.

Participants' follow-up (risk time and deaths) was first split into intervals of 6 months by current age ( $0-100$ years), calendar time (1997-2011 incl.) and diabetes duration (020 years). Risk time and deaths (overall and by cause) were subsequently tabulated by current age, date of follow-up and diabetes duration, and each cell of the table was assigned age, date and diabetes duration as continuous variables as the midpoint of the 6 month group. Age at diagnosis was calculated as current age minus diabetes duration. Data were analysed using a Poisson model, using log-person-time as the offset variable and spline effects of current age, diabetes duration and age at diagnosis, and with a linear effect of calendar time. The model is formally over-parametrised because age at diagnosis plus diabetes duration equals current age. Therefore, it is not possible to tease out the separate effects of age at diabetes diagnosis and diabetes duration. We only used the model for prediction of rates, and these do not depend on any particular parametrisation. Separate models were fitted for men and women.

The predicted mortality rates by current age for individuals diagnosed at different ages are shown in Fig. 1. To illustrate the effect of age at diagnosis and duration of type 2 diabetes, we computed the mortality rate ratio (also known as HR) between individuals diagnosed at ages 5 years apart as a function of age at follow-up (e.g. age 40 vs 45 at diagnosis, followed from age 45 to 60 ). Thus, each point on each curve indicates the mortality risk for a person diagnosed at one age compared with a person diagnosed at 5 years older, when both individuals have achieved the same age. Different curves were used to illustrate how this effect varies by age at diagnosis, by comparing individuals diagnosed at ages 45 vs 50,50 vs 55 , etc. The same was done for a 10 year difference in diabetes duration, by comparing individuals diagnosed at ages 40 vs 50,45 vs 55, etc. The two sets of curves are shown in adjacent graphs in Fig. 2.

On the basis of the underlying cause of death for people with type 2 diabetes, we carried out the same analyses for cause-specific mortality from CVD, cancer and other causes of death.

We report results on two populations: the primary population is that for which age of diabetes diagnosis is available $(n=744,188)$. For this analysis, after excluding 479 registrants, because the registration date was the same as the date of death or the dates were implausible, the sample size was 743,709 . However, given that only $67 \%$ of the 
Fig. 1 Predicted (a) all-cause mortality, (b) CVD mortality, (c) cancer mortality and (d) nonCVD, non-cancer mortality rate, as a function of current age for individuals with diabetes.

Separate curves are shown for separate ages at diabetes diagnosis (ages 40, 45, 50, 55, 60, 65 and 70). The model used for prediction included non-linear effects of current diabetes duration and age as well as age at diagnosis. A linear effect of calendar time was also included in the model; the curves refer to 1 January 2010. Blue lines, men; red lines, women; dotted lines, 95\% CI
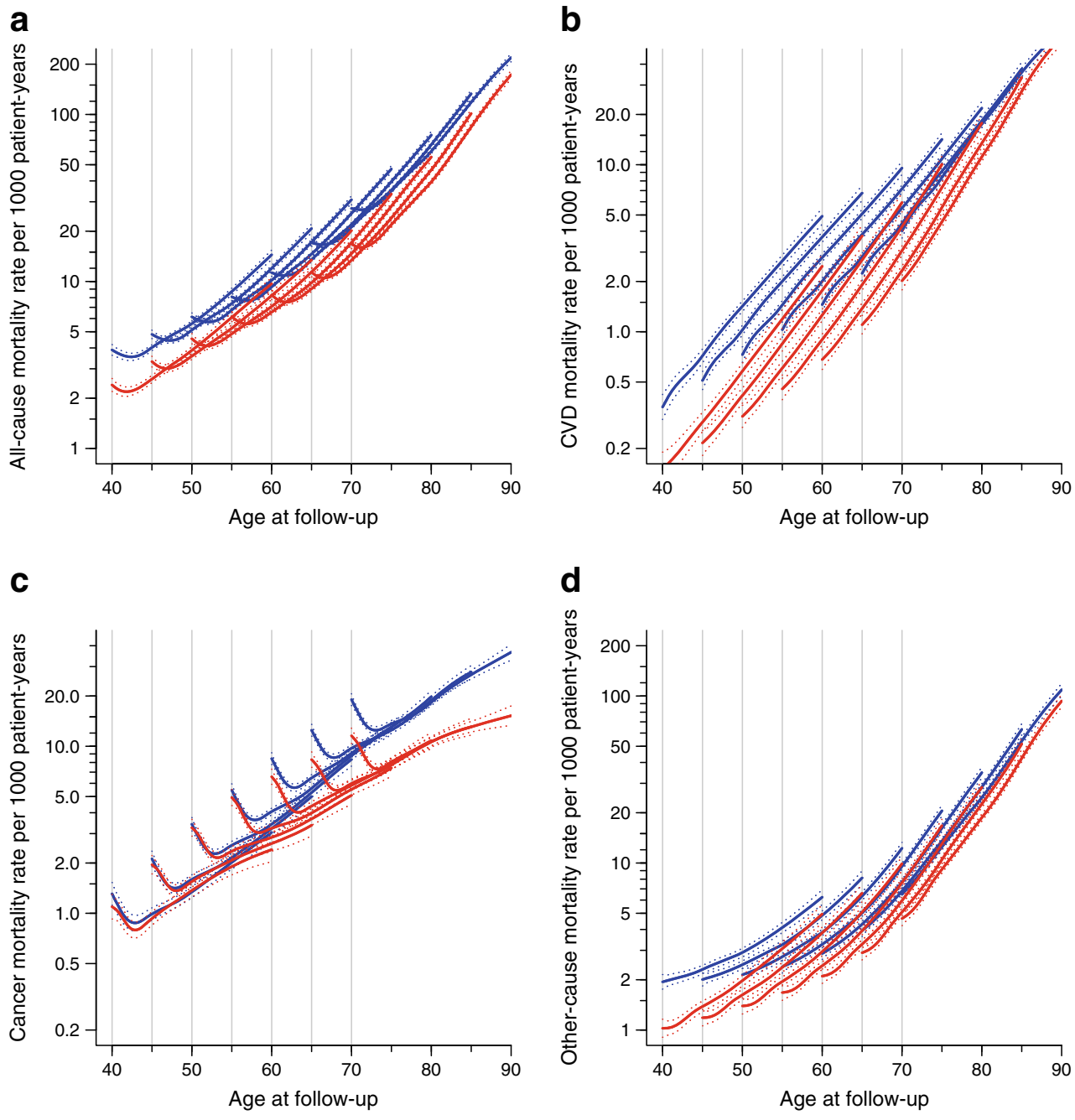

entire NDSS type 2 diabetes cohort over the time period 1997-2011 had the date of diabetes recorded, the analysis was repeated for all registrants using the registration date as a proxy for those with a missing date of diagnosis $(n=$ 1,108,420). After excluding 743 registrants because the registration date was the same as the date of death, or the dates were implausible, the sample size for the sensitivity analysis was 1,107,677.

All analyses and graphs were generated with $\mathrm{R}$ software, version 3.4 .2 (www.R-project.org) (R Foundation for Statistical Computing, Vienna, Austria), using packages Epi and popEpi. The study was approved by the Alfred Health Human Ethics Committee and the Australian Institute of Health and Welfare Ethics Committee. All participants gave informed consent for their data to be used in the analyses. A complete account of the data analyses can be found at http://bendixcarstensen.com/IDI/ mort/AUS-DM-mort.pdf. The data that support the findings of this study are not publicly available as they were generated under a licence which restricted access to named, approved investigators.

\section{Results}

Study population characteristics Table 1 describes the characteristics of the 743,709 participants who had a date of diagnosis of diabetes (primary analysis cohort) and the full cohort including those for whom registration was used as a proxy for the missing age of diagnosis date $(n=1,107,677)$. In both groups, the median age at registration was about 60 years, $54 \%$ of registrants were men and the median follow-up time was 7.2 years. In the primary dataset, the median age at diagnosis was 58.6 years (interquartile range [IQR] 49.4-67.9) while in the full dataset, the median age at diagnosis was 59.6 years (interquartile range [IQR] 50.2-68.9). The mortality rate was higher in the full cohort than in the primary analysis cohort (26.5 vs 20.9 per 1000 patient-years).

The following results refer to the analysis of individuals with a known date of diagnosis of type 2 diabetes.

All-cause mortality rates All-cause mortality rates by age and sex for people with diabetes diagnosed at ages 40, 45, 50, 55 through to 70 years are shown in Fig. 1a. The all-cause 
a

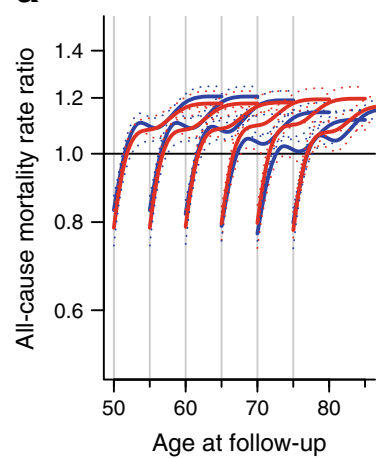

e

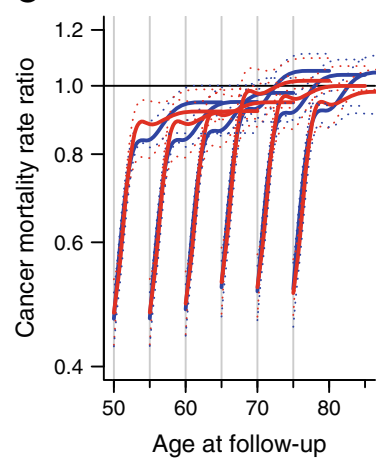

b

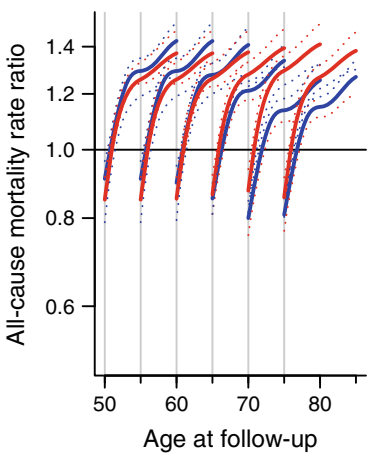

f

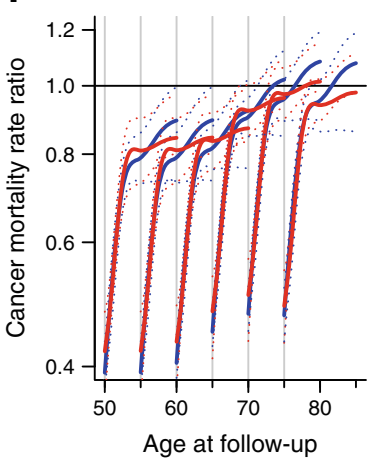

C

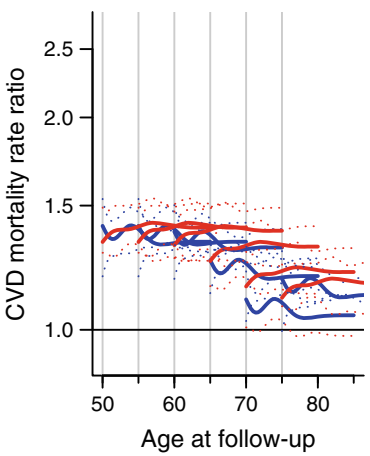

g

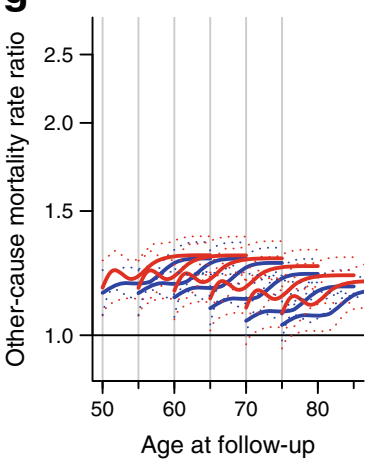

d

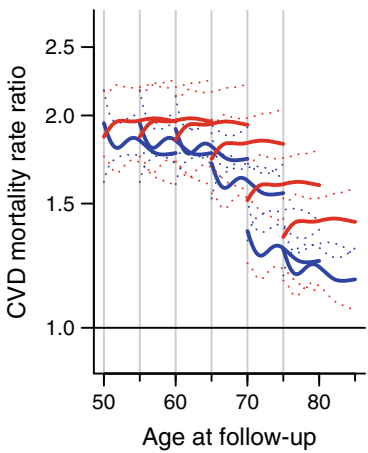

h

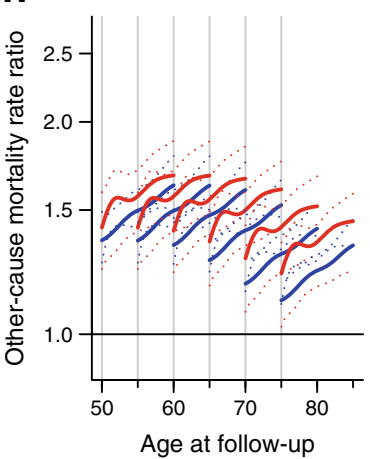

Fig. 2 Mortality rate ratios for younger onset vs older onset (corresponding to longer vs shorter duration) of diabetes. (a, c, e, g) Mortality rate ratios between diabetic individuals of the same age but diagnosed 5 years apart and hence with 5 years' longer duration of diabetes at any age. The leftmost pair of curves in each graph is the mortality rate ratio between people diagnosed at ages 45 and 50 , over the 15 year period from age 50 to 65 . The next pair shows the mortality rate ratio between people diagnosed at ages 50 and 55, as they age from 55 to 70 years old. Moving to the right, curves show the mortality rate ratios between those diagnosed at 55 and 60, 60 and 65, and 70 and 75. The curves thus represent how the mortality rate ratio associated with a 5 year difference in duration of diabetes varies by age and diabetes duration. The differences between the curves show how the effect of a 5 year difference in duration depends on age at diagnosis. (b, d, f, h) Similar curves, except for individuals diagnosed 10 years apart, and hence with 10 years' longer duration, showing the effect of a 10 year longer duration of diabetes, comparing

mortality rates declined during the first 1-2 years after diabetes diagnosis and then increased exponentially with age (Fig. 1a). At any given age, all-cause mortality rates were higher for a lower age of diagnosis (therefore longer duration) in both men and women. The all-cause mortality rate ratio for a 5 year difference in age at diagnosis (the younger age at diagnosis vs the older age at diagnosis) started below 1.0 but increased to around 1.2-1.3 during the first 6 years after diagnosis (Fig. 2a). The same pattern was apparent when using a 10 year difference in age at diagnosis (Fig. 2b). Figure $2 \mathrm{a}, \mathrm{b}$ also shows that as age at diagnosis increased, the excess mortality risk of earlier disease onset (corresponding to longer duration) was largely constant among women but decreased among men. The excess risk associated with a 10 year difference of durations 10-20 years with those of 0-10 years (since we only have reliable data to 20 years of duration). The leftmost curves in each graph show the mortality rate ratio between people diagnosed at ages 40 and 50 . Moving to the right, the curves show the rate ratios for people diagnosed at 45 and 55,50 and 60,55 and 65,60 and 70, and 65 and 75. The curves in this figure thus represent the joint effect of age at diagnosis and duration of diabetes by comparing successive curves in Fig. 1. (a) All-cause mortality and 5 years' difference in age of diabetes onset. (b) All-cause mortality and 10 years' difference in age of diabetes onset. (c) CVD mortality and 5 years' difference in age of diabetes onset. (d) CVD mortality and 10 years' difference in age of diabetes onset. (e) Cancer mortality and 5 years' difference in age of diabetes onset. (f) Cancer mortality and 10 years' difference in age of diabetes onset. (g) Non-CVD, noncancer mortality and 5 years' difference in age of diabetes onset. (h) NonCVD, non-cancer mortality and 10 years' difference in age of diabetes onset. Blue lines, men; red lines, women; dotted lines, 95\% CI

age was (not surprisingly) greater than that associated with a 5 year difference.

CVD mortality rates increased exponentially with age in both sexes and across all groups with different ages at diagnosis of diabetes (Fig. 1b). At any given age, CVD mortality also increased with decreasing age at diagnosis (corresponding to increasing duration) of diabetes. Between two men aged 50 years and diagnosed with diabetes at ages 45 and 50 years, i.e. with 5 years' difference in diabetes duration, the CVD mortality risk was approximately 1.4 times as high in the one diagnosed at the younger age (Fig. 2c, d). This excess risk fell by age at diagnosis but remained over 1.0 (Fig. 2c, leftmost curve). As the age of diagnosis increased (e.g. $45 \mathrm{vs}$ 50 , compared with 55 vs 60 ), the excess risk associated with 
Table 1 Characteristics of type 2 diabetic individuals on the NDSS between 1997 and 2011

\begin{tabular}{|c|c|c|c|c|c|c|}
\hline & \multicolumn{3}{|c|}{$\begin{array}{l}\text { Primary analysis cohort } \\
\text { (population with age of diagnosis data) }\end{array}$} & \multicolumn{3}{|c|}{$\begin{array}{l}\text { Full cohort (population where missing } \\
\text { age of diagnosis data was replaced with } \\
\text { date of registration) }\end{array}$} \\
\hline & Men & Women & Total & Men & Women & Total \\
\hline No. people, $n(\%)$ & $406,277(54.6)$ & $337,432(45.4)$ & 743,709 & $599,257(54.1)$ & $508,420(45.9)$ & $1,107,677$ \\
\hline $\begin{array}{l}\text { Age at registration } \\
\text { years median (IQR) }\end{array}$ & $59.7(51.0-68.6)$ & $60.7(50.8-70.6)$ & $60.2(50.9-69.5)$ & $60.3(51.5-69.0)$ & $61.2(51.1-71.0)$ & $60.7(51.3-69.9)$ \\
\hline $\begin{array}{l}\text { Age at diagnosis } \\
\text { years median (IQR) }\end{array}$ & $58.2(49.4-67.0)$ & $59.1(49.3-68.9)$ & $58.6(49.4-67.9)$ & $59.2(50.4-68.0)$ & $60.1(50.0-70.0)$ & $59.6(50.2-68.9)$ \\
\hline $\begin{array}{l}\text { Follow-up time } \\
\text { years median (IQR) }\end{array}$ & $6.9(3.6-11.0)$ & $7.5(3.6-11.6)$ & $7.2(3.4-11.3)$ & $6.8(3.1-11.8)$ & $7.6(3.5-12.8)$ & $7.2(3.3-12.3)$ \\
\hline $\begin{array}{l}\text { Mortality, } n(95 \% \mathrm{CI}) \\
\text { per } 1000 \text { patient-years }\end{array}$ & $22.8(22.6,22.9)$ & $18.8(18.6,19.0)$ & $20.9(20.8,21.0)$ & $28.7(28.5,28.8)$ & $24.1(23.9,24.2)$ & $26.5(26.4,26.6)$ \\
\hline $\begin{array}{l}\text { Total number of deaths } \\
\text { during follow-up }\end{array}$ & 66,987 & 48,376 & 115,363 & 128,034 & 97,274 & 225,308 \\
\hline \multicolumn{7}{|l|}{ Cause-specific deaths, $n(\%)$} \\
\hline CVD & $18,978(28.3)$ & $13,716(28.4)$ & $32,694(28.3)$ & $40,079(31.3)$ & $30,606(31.5)$ & $70,685(31.4)$ \\
\hline IHD & $14,589(21.8)$ & $9297(19.2)$ & $23,886(20.7)$ & $30,708(24.0)$ & $20,986(21.6)$ & $51,694(22.9)$ \\
\hline Stroke & $4389(6.6)$ & $4419(9.1)$ & $8808(7.6)$ & $9371(7.3)$ & $9620(9.9)$ & $18,991(8.4)$ \\
\hline Cancer & $22,430(33.5)$ & $13,577(28.1)$ & $36,007(31.2)$ & $37,285(29.1)$ & $22,654(23.3)$ & $59,939(26.6)$ \\
\hline Pancreatic cancer & $2378(3.5)$ & $1912(4.0)$ & $4290(3.7)$ & $3524(2.8)$ & $2820(2.9)$ & $6344(2.8)$ \\
\hline Lung cancer & $4448(6.6)$ & $1961(4.1)$ & $6409(5.6)$ & $7374(5.8)$ & $3217(3.3)$ & $10,591(4.7)$ \\
\hline Colorectal cancer & $2186(3.3)$ & $1314(2.7)$ & $3500(3.0)$ & $3797(3.0)$ & $2337(2.4)$ & $6134(2.7)$ \\
\hline Non-CVD, non-cancer & $25,579(38.2)$ & $21,083(43.6)$ & $46,662(40.4)$ & $50,670(39.6)$ & $44,014(45.2)$ & $94,684(42.0)$ \\
\hline
\end{tabular}

diagnosis at a younger age was smaller but still apparent. Similar patterns were seen in women, though the excess risks of earlier-onset diabetes were slightly higher among women than among men (Fig. 2c). A similar pattern was seen for 10 years' difference in duration (equalling 10 years' difference in age at diagnosis) but at a higher relative difference (Fig. 2d).

Both IHD mortality and stroke mortality increased with age. At any given age, IHD and stroke mortality rates were higher for a lower age of diagnosis (and so longer duration) in both men and women (ESM Fig. Kn-5, Kn-6).

The cancer mortality rates were high just after diagnosis, declined during the first 3 years, and then increased with age (Fig. 1c). The effect of age at diagnosis on cancer mortality was opposite to that observed for CVD and all-cause mortality, such that, at any given age, the earlier age at diagnosis was associated with lower cancer mortality (Figs 1c 2e, 2f) and was most pronounced among women. We also analysed three other cancer mortality outcomes: pancreatic cancer, lung cancer and colorectal cancer (ESM Fig. Kn-7-9). We observed a sharp increase in pancreatic cancer mortality shortly after diabetes diagnosis, which decreased after 3 years, consistent with reverse causation. The observed pattern is consistent with no effect of duration or age at diagnosis on mortality (ESM Fig. Kn-9). For lung cancer, earlier onset of diabetes was associated with lower lung cancer mortality (ESM Fig. Kn-7). For colorectal cancer, there was no effect of age of diabetes onset among men, but among women there was a slightly reduced colorectal cancer mortality for those with younger-onset diabetes (ESM Fig. Kn-8).

For other causes of death (non-CVD, non-cancer), rates increased with increasing age, and younger age of diabetes onset was associated with increased non-CVD, non-cancer mortality (Fig. 1d). For two people of the same age, but with 5 years' difference in diabetes onset, the person with the younger onset of diabetes had an approximately 25-30\% higher non-CVD, non-cancer mortality risk relative to the person with the older age of onset, at any age. This elevated risk decreased with increasing age of diagnosis (Fig. 2g, h). For two individuals at the same age but with 10 years' difference in diabetes onset, the person with the younger onset of diabetes had an approximately 40-50\% higher non-CVD, noncancer mortality risk relative to the person with the older age at onset, but the effect decreased with increasing age.

In sensitivity analyses, we repeated the entire analysis on our secondary population ( $n=1,107,677)$ using registration date as a proxy for date of diabetes diagnosis. We observed similar patterns to those seen using the cohort who had complete information with regard to age of diagnosis of diabetes.

\section{Discussion}

Using a population-based cohort including approximately 90\% of all Australians with type 2 diabetes between 1997 
and 2011, we showed that a younger age at diagnosis of type 2 diabetes was associated with higher all-cause and CVD, IHD and stroke mortality, but slightly lower cancer mortality (allcause and lung and colorectal cancers), compared with diagnosis at an older age. To put these results into context, for two men of the same age but with 10 years' difference in diabetes duration, the one with the earlier onset (and hence longer duration of diabetes) had an approximately $30 \%$ and $60 \%$ greater chance of death from any cause and from CVD, respectively, relative to the man with the shorter duration, at any age.

Only a limited number of studies have examined the effect of age at diagnosis on risk of mortality. In studies where this has been examined most have used age at diagnosis or diabetes duration as a categorical rather than a continuous variable. A recent study from Denmark [11] showed that, at any given age, younger age at diagnosis of type 2 diabetes was associated with higher all-cause mortality for men, but the excess risk attributable to age at diagnosis was only very weak among women. This work only examined all-cause mortality and was clinic-based, in contrast to our findings which showed similar significant relationships in men and women. Using population data, our work assessed how long-term overall and causespecific mortality depended on current age, age at diagnosis and diabetes duration in people with type 2 diabetes, and extended these findings. In a unique approach we demonstrated that younger age at diagnosis was consistently associated with higher all-cause and CVD mortality (relative to older age at diagnosis) across the range of age at diagnosis and in both men and women in the entire Australian diabetic population. Even though individuals with early diabetes onset had been subject to mortality selection for a period, they appeared to maintain a higher mortality when compared with those with later onset, due to a combination of the effect of early age at onset and the effect of duration of type 2 diabetes; however, these two effects cannot be separated.

Our primary analyses were conducted among the group with a known date of diagnosis. When we undertook the analyses in the full cohort, the results were very similar to those from the cohort with age of diabetes diagnosis data. The characteristics of these two populations were similar except for a higher crude mortality rate in the full cohort compared with the primary analysis cohort (restricted to those with a date of diabetes diagnosis).

It is not fully understood why age at diagnosis and duration of diabetes are related to mortality. A substantial component of the excess risk in younger-onset diabetes is likely to be due to the longer duration of diabetes, which clearly increases the exposure to hyperglycaemia. Since age is such an important driver of mortality, it was essential in our analysis to fix the current age when comparing mortality outcomes of different ages of onset, meaning that a 5 year earlier age of onset also meant a 5 year longer duration of diabetes. However, we also saw that for CVD mortality and for non-CVD, non-cancer mortality the effect of a 5 or 10 year earlier age of onset was greater in younger than in older people. This may be because in younger people there are fewer additional risk factors, and so the impact of hyperglycaemia is greater than among older people, in whom a range of other risk factors is typically also present.

Evidence is accumulating to suggest that earlier onset of type 2 diabetes is associated with an increased risk of complications and comorbidities compared with later onset, and that the development and progression of complications might be more aggressive in those with earlier onset. In Pima Indians, youth-onset type 2 diabetes is associated with a similar frequency of overt nephropathy [18] but a substantially increased incidence of end-stage renal disease compared with adultonset type 2 diabetes [19]. Further, in this cohort, retinopathy risk at 20 years' diabetes duration was higher for youth-onset than for older-onset type 2 diabetes despite the younger current age. Thus, in contrast to mortality outcomes, for microvascular outcomes the influence of age is not as strong as it is for diabetes duration.

Findings from other studies also show that those with earlyonset diabetes have worse CVD profiles than those diagnosed at older ages. A cross-sectional study conducted in a primary care setting showed that those with early-onset type 2 diabetes in the UK (mean age 34 years) already had a cardiovascular risk profile (overweight, hyperlipidaemia, hypertension) similar to that of an older adult with type 2 diabetes (mean age 67 years) [20]. Those with early-onset type 2 diabetes are also more likely to have poorer glucose control, untreated hypertension and hyperlipidaemia [21]. Given this, there have been several studies which have tried to intervene in such individuals. The TODAY study showed that even with the resources to encourage consistent early lifestyle modification and the provision of medications for glycaemic control and aggressive ACE inhibitor therapy, the prevalence of both hypertension and microalbuminuria increased markedly over time among adolescents with type 2 diabetes regardless of diabetes treatment [6]. More recently a small study from Australia showed that those with diabetes who were diagnosed between 15 and 30 years of age had more severe albuminuria and neuropathy scores compared with those diagnosed at 40-50 years of age after adjustment for diabetes duration [22]. As such, increased clinical attention is imperative for individuals with earlieronset type 2 diabetes. Efforts should focus on timely optimisation of individuals' self-management skills and medical treatment to prevent or reduce the onset of complications and comorbidities. Additionally, there is a need to identify and screen those at high risk of developing diabetes so that individuals can make lifestyle changes that will prevent or delay the onset of diabetes.

We observed higher relative mortality in people with recently diagnosed diabetes ( $<2$ years) than for people with 
moderate diabetes duration, which has also been reported in other studies [23, 24]. This is most likely because people diagnosed with diabetes because of other medical conditions (e.g. cancer) are over-represented among newly diagnosed individuals and are more likely to die in the first short period after diagnosis of diabetes.

The link between type 2 diabetes and cancer has been consistently documented for a large array of different cancer sites and irrespective of the study design [25-27]. Interestingly, we found that earlier age at diagnosis of diabetes seemed to be related to lower cancer mortality, which was in contrast to CVD mortality. It is possible that following a diagnosis of diabetes, people have more frequent contact with the healthcare system, which may increase the likelihood of any present but undiagnosed cancer being detected. Our previous study showed that cancer incidence was significantly higher in the first 3 months after registration on the NDSS, suggesting the presence of detection bias and/or reverse causation among newly diagnosed type 2 diabetic individuals [16]. Similarly, we demonstrate this in the current analysis with mortality from pancreatic cancer. This may contribute to better cancer survival for individuals with earlier age at diagnosis of diabetes.

Strengths and limitations The main strength of this study was the use of population-based administrative data with a large sample size and long follow-up time. The results are therefore unlikely to be confounded by data collection procedures. Hence, our results are likely to be generalisable to Western countries similar to Australia.

A key weakness is that the NDSS lacks information on potentially important covariates, such as ethnicity, smoking status, weight or BMI, glycaemic control, physical activity, treatment and comorbidities.

In conclusion, occurrence of type 2 diabetes at an earlier age places individuals at increased risk of premature death, especially from CVD. Early and aggressive risk factor management is warranted for individuals with young-onset type 2 diabetes.

Acknowledgements We thank the NDSS, an initiative of the Australian government administered by Diabetes Australia, for the data source and thank the Australian Institute of Health and Welfare for the linkage of the NDSS to the National Death Index.

Data availability The data that support the findings of this study are not publicly available as they were generated under a licence for a large linkage project, which restricted access to named, approved investigators.

Funding This study was funded by the National Health and Medical Research Council (NHMRC Grant APP 1002663) and was supported in part by the Victorian government's Operational Infrastructure Support Program. LH is supported by the China Scholarship Council and Beijing Municipal Administration of Hospitals' Youth Program (QML20160404). JES and DJM are supported by NHMRC Senior Research Fellowships.
Duality of interest The authors declare that there is no duality of interest associated with this manuscript.

Contribution statement LH made substantial contributions to the conception and design of the work and to data analysis and interpretation, and initially drafted and revised the manuscript critically for important intellectual content. DJM made substantial contributions to the conception and design of the work and to data acquisition, analysis and interpretation, and revised the manuscript critically for important intellectual content. JLH made a substantial contribution to data acquisition and interpretation and revised the manuscript critically for important intellectual content. FR and $\mathrm{NN}$ made a substantial contribution to interpretation of the data and revised the manuscript critically for important intellectual content. JES made substantial contributions to the conception and design of the work and to data acquisition and interpretation, and revised the manuscript critically for important intellectual content. BC made substantial contributions to the conception and design of the work, conducted the main data analysis, contributed to the interpretation of data, and revised the manuscript critically for important intellectual content. All authors approved the final version of the article. DJM and $\mathrm{BC}$ are the guarantors of this work and, as such, had full access to all the data in the study and take responsibility for the integrity of the data and the accuracy of the data analysis.

\section{References}

1. Kitagawa T, Owada M, Urakami T, Yamauchi K (1998) Increased incidence of non-insulin dependent diabetes mellitus among Japanese schoolchildren correlates with an increased intake of animal protein and fat. Clin Pediatr 37:111-115

2. Australian Institute of Health and Welfare (2014) Type 2 diabetes in Australia's children and young people: a working pape. In: Diabetes Series Australian Institute of Health and Welfare, Canberra

3. Grinstein G, Muzumdar R, Aponte L, Vuguin P, Saenger P, DiMartino-Nardi J (2003) Presentation and 5-year follow-up of type 2 diabetes mellitus in African-American and CaribbeanHispanic adolescents. Horm Res 60:121-126

4. Dabelea D, DeGroat J, Sorrelman C et al (2009) Diabetes in Navajo youth: prevalence, incidence, and clinical characteristics: the SEARCH for Diabetes in Youth Study. Diabetes Care 32(Suppl 2):S141-S147

5. Craig ME, Femia G, Broyda V, Lloyd M, Howard NJ (2007) Type 2 diabetes in Indigenous and non-Indigenous children and adolescents in New South Wales. Med J Aust 186:497-499

6. TODAY Study Group (2013) Rapid rise in hypertension and nephropathy in youth with type 2 diabetes: the TODAY clinical trial. Diabetes Care 36:1735-1741

7. TODAY Study Group (2013) Lipid and inflammatory cardiovascular risk worsens over 3 years in youth with type 2 diabetes: the TODAY clinical trial. Diabetes Care 36:1758-1764

8. West NA, Hamman RF, Mayer-Davis EJ et al (2009) Cardiovascular risk factors among youth with and without type 2 diabetes: differences and possible mechanisms. Diabetes Care 32: 175-180

9. Rodriguez BL, Fujimoto WY, Mayer-Davis EJ et al (2006) Prevalence of cardiovascular disease risk factors in U.S. children and adolescents with diabetes: the SEARCH for Diabetes in Youth Study. Diabetes Care 29:1891-1896

10. Hillier TA, Pedula KL (2003) Complications in young adults with early-onset type 2 diabetes: losing the relative protection of youth. Diabetes Care 26:2999-3005

11. Faerch K, Carstensen B, Almdal TP, Jorgensen ME (2014) Improved survival among patients with complicated type 2 diabetes 
in Denmark: a prospective study (2002-2010). J Clin Endocrinol Metab 99:E642-E646

12. Harding JL, Shaw JE, Peeters A, Guiver T, Davidson S, Magliano DJ (2014) Mortality trends among people with type 1 and type 2 diabetes in Australia: 1997-2010. Diabetes Care 37:2579-2586

13. Tancredi M, Rosengren A, Svensson AM et al (2015) Excess mortality among persons with type 2 diabetes. N Engl J Med 373:1720 1732

14. Chang CH, Shau WY, Jiang YD et al (2010) Type 2 diabetes prevalence and incidence among adults in Taiwan during 1999-2004: a national health insurance data set study. Diabet Med 27:636-643

15. Loh V, Harding J, Koshkina V, Barr E, Shaw J, Magliano D (2014) The validity of self-reported cancer in an Australian population study. Aust N Z J Public Health 38:35-38

16. Harding JL, Shaw JE, Peeters A, Cartensen B, Magliano DJ (2015) Cancer risk among people with type 1 and type 2 diabetes: disentangling true associations, detection bias, and reverse causation. Diabetes Care 38:264-270

17. Harding J, Shaw JE, Peeters A, Davidson S, Magliano DJ (2016) Age-specific trends from 2000-2011 in all-cause and cause-specific mortality in type 1 and type 2 diabetes: a cohort study of more than one million people. Diabetes Care 39:1018-1026

18. Krakoff J, Lindsay RS, Looker HC, Nelson RG, Hanson RL, Knowler WC (2003) Incidence of retinopathy and nephropathy in youth-onset compared with adult-onset type 2 diabetes. Diabetes Care 26:76-81

19. Pavkov ME, Bennett PH, Knowler WC, Krakoff J, Sievers ML, Nelson RG (2006) Effect of youth-onset type 2 diabetes mellitus on incidence of end-stage renal disease and mortality in young and middle-aged pima Indians. JAMA 296:421-426

20. Gunathilake W, Song S, Sridharan S, Fernando DJ, Idris I (2010) Cardiovascular and metabolic risk profiles in young and old patients with type 2 diabetes. QJM 103:881-884

21. Song SH, Hardisty CA (2009) Early onset type 2 diabetes mellitus: a harbinger for complications in later years-clinical observation from a secondary care cohort. QJM 102:799-806

22. Al-Saeed AH, Constantino MI, Molyneaux L et al (2016) An inverse relationship between age of type 2 diabetes onset and complication risk and mortality: the impact of youth-onset type 2 diabetes. Diabetes Care 39:823-829

23. Carstensen B, Kristensen JK, Ottosen P, Borch-Johnsen K, Steering Group of the National Diabetes Register (2008) The Danish National Diabetes Register: trends in incidence, prevalence and mortality. Diabetologia 51:2187-2196

24. Walker JJ, Livingstone SJ, Colhoun HM et al (2011) Effect of socioeconomic status on mortality among people with type 2 diabetes: a study from the Scottish Diabetes Research Network Epidemiology Group. Diabetes Care 34:1127-1132

25. Barone BB, Yeh HC, Snyder CF et al (2008) Long-term all-cause mortality in cancer patients with preexisting diabetes mellitus: a systematic review and meta-analysis. JAMA 300:2754-2764

26. Coughlin SS, Calle EE, Teras LR, Petrelli J, Thun MJ (2004) Diabetes mellitus as a predictor of cancer mortality in a large cohort of US adults. Am J Epidemiol 159:1160-1167

27. Verlato G, Zoppini G, Bonora E, Muggeo M (2003) Mortality from site-specific malignancies in type 2 diabetic patients from Verona. Diabetes Care 26:1047-1051 